We had published the following article in Volume 9, Issue 40.

\title{
Doses Delivered to Patients and Associated Risks from Conventional Radiological Scans in Yasuj City, Iran
}

Amin Hassanvand ${ }^{1}$, Hamid Reza Masjedi², Hassan Vafapure ${ }^{3}$, Hamed Zamani ${ }^{4}$, Shiva Rahbar Yazdi ${ }^{5}$, Mohammad Hossein Zare ${ }^{6}$

The above article has been withdrawn on authors' request.

Hassanvand A, Masjedi HR, Zamani $\mathrm{H}$, et al. Doses delivered to patients and associated risks from conventional radiological scans in Yasuj city, Iran. J Evolution Med Dent Sci 2020;9(40):2997-3003, DOI: 10.14260/jemds/2020/656

\section{Citation for Retraction-}

J Evolution Med Dent Sci 2020;10(44): Page Number 3815. New, DOI: 10.14260/jemds/2021/771 